\title{
Bio-based design methodologies for products, processes, machine tools and production systems
}

\author{
van Houten, F.a †, Wertheim, R.b, ${ }^{*}$, Ayali, A.e, Poverenov, E.c, Mechraz, G.c, Eckert, U.d, Rentzsch, H.d, Dani, I.d, \\ Willocx, M. f, Duflou, J.R.f, g,
}

a UT, University of Twente, The Netherlands, † Oct 18th 2020

b Fraunhofer IWU, Chemnitz, Germany \& Ort Braude College, Karmiel, Israel

c ARO, Volcani Institute, Israel

d Fraunhofer Institute for Machine Tools and Forming Technology, Chemnitz, Germany

e TAU, Tel Aviv University, Israel

f KU Leuven, Belgium

g Member of Flanders Make

*Corresponding Author: Wertheim, R.; wertheim@braude.ac.il ; Tel +972544660658

\begin{abstract}
The paper explores and presents results of an investigation handling design methodologies using the bio-space and biological phenomena combined with the technical space for the development, design and application of products, machine tools, processes and manufacturing systems carried out by a multinational team including Fraunhofer and CIRP fellows. The main biological approach for designing was bio-inspiration. However, links and examples implementing bio-integration and bio-intelligence were also considered and analyzed during the investigation.

The paper describes a systematic design procedure starting from requirements and applications related to manufacturing, using well-known theories and experience in engineering, however analyzing and applying biological materials, properties, structures, phenomena and complete systems. Sustainability aspects combined with the immense and ever growing capacity of ICT and digitization possibilities provided new options for design methodologies.

A moveable and flexible machine tool was virtually used as a demonstrator to describe the new methodology. It was proved that the o ngoing process of learning from nature and applying biological phenomena, structures and materials, together with new technologies such as Additive Manufacturing (AM) can develop new ideas, processes, manufacturing systems, and products with benefits to performance, productivity, efficiency and sustainability. Nature and biological domains can be integrated, can inspire, be implemented or even applied as an intelligent system in the design procedure.
\end{abstract}

Keywords: Biologicalization, Biological Approach, Bio-inspiration, Bio-intelligence, Design Methodologies, Manufacturing Systems

\section{1}

\section{Introduction - goals and challenges}

Design topics were recently presented by authors of this paper in the CIRP journal (2018) under the title "Biologicalisation: Biological transformation in manufacturing", discussing the inter relations between nature and technology [1]. Bio-inspiration can serve designers in two distinct ways: As a trigger for searching and implementing a solution for an engineering problem or as a trigger by using observation in the bio-space to be converted or adapted to the technical space. The current paper is emphasizing new design methodologies, describing systematical design procedures, using examples from biology, (bottom up) and engineering (top down) as well as IT technologies.

A broad survey on design methodologies used in industrial and educational applications was published and presented as a keynote paper in CIRP in 2009 [2].The overview of Design Theory and Methodology (DTM) described here came to the conclusion that design is based on mathematics and concrete goals, and on process technologies. However, the methodologies that do not emphasize innovative design are not very useful for advanced and complex designs, or for improving a known design. Although fundamental concepts and organized knowledge related to product development are well-known, achieving innovative design requires data regarding complexity, multi-disciplinarily, integration of ICT, globalization, virtual engineering, and collaboration with stakeholders from different cultural and educational backgrounds. The keynote paper did not discuss sustainability aspects combined with the use of biological resources, principles and bio-intelligence, based on learning from nature and new technologies such as $\mathrm{AM}$, but these aspects were introduced in few CIRP papers during the last decade $[3,4,5]$. Bioinspiration of surfaces and textures are discussed in $[6,7]$ and further publications $[8,9]$ combine bio-inspiration with topics such as DNA and Additive Manufacturing. The growing need to include ever more sustainability issues in design increases the complexity of the design process and of its outcome (the designed item). Fortunately, the immense and ever-growing capacity of IT and digitization possibilities provide inspiring options for new, more effective design methodologies.

In manufacturing, topics of bio-integration, bio-inspiration and bio-intelligence are not new, although design has been lacking a systematic approach. A scientifically based method for capturing knowledge must be developed in order to achieve a consistent mapping of biological principles on design rules for the creation of artefacts such as products, machine tools and processes.

The bio-inspired paradigm of effectivity by redundancy is enabled by digitization in the set-based design. The design is combined by considering sustainability as a major boundary condition (constraint) for the search space from the beginning. In the classical approach of optimization this usually is one of the last items to be addressed. The following institutes and research topics were included in the multinational teamwork carrying out the investigation on bio-based design methodologies for manufacturing:

- A systematical computer-based synthesis/analysis was carried out by the University of Twente in the Netherlands,

- A systematic analytical data collection method was carried out by KU Leuven in Belgium

- Gathering information on the development of modern machine tools and up-to-date manufacturing systems such as EDM was carried out by Fraunhofer IWU, Germany,

- Up-to-date knowledge on investigations regarding biological principles and materials, including coatings, was supplied by 
the Tel Aviv University and Volcani Institute in Israel, respectively.

A moveable and flexible machine tool was used as a virtual demonstrator to describe the new methodology. Several required features and physical properties for the design of this machine tool were defined and a top down systematical biological procedure was used. The multinational team analyzed and applied, for the development of the moveable and flexible machine tool, a systematic computer-based analysis, the experience and development knowledge of modern machine tools, a statistical and analytical data collection, as well as new up-to-date biological knowledge and investigation results.

The main goal of this investigation was to link bio-inspired solution principles to constraint-based design support systems and to prominently include sustainability issues: Eco footprint, energy efficiency, lightweight components, de-materialization etc. This includes extending the concept of industry 4.0 (Simulation: Digital Twins, agility, adaptability etc.) with principles from biology in order to support the design of products and machines with life-like behavior.

The major challenge was to prove that an ongoing process of learning from nature and using biological phenomena, structures and materials, together with new technologies, such as AM, can provide new ideas, processes, manufacturing systems, and products with benefits to performance, productivity, efficiency, and sustainability.

\section{Design and application of bio-inspired surfaces,} components, machines and systems

Design of bio-inspired surfaces, components, machines and systems for manufacturing and application is not new, but has been developed and applied more frequently $[1,4,5,8,9]$. However, bio-inspiration, including bio-integration in design, is lacking a systematic approach, a scientific use of biological data and an efficient use of ICT resources. A scientifically based method using new data resources and new technologies for capturing knowledge must be developed in order to achieve consistent mapping of biological principles influencing design rules for the creation of artefacts such as products, machine tools and processes.

For many years, designers have been educated and trained to consider the constraints of production processes as the most important guidelines for their design decisions (DfX: Design for Manufacturing, Design for Assembly, Design for Use, etc.). New manufacturing techniques and new materials create much more freedom for designers to combine complex geometries with localized material behavior. New modeling techniques enable better prediction of the behavior of products and production systems. This opens up the possibility for a giant, transformational leap to "Manufacturing for Design", enabling more complex, smaller and lightweight geometries and structures. It will even improve the possibility to integrate sensors and actuators.

During the first industrial revolution, design was prominently a discipline in the mechanical domain. That changed in the second industrial revolution when electro-mechanical systems were developed, and once again in the third industrial revolution when computers were introduced. Software development became an integral part of the design discipline. Another important change comprised the extension of the designers' responsibilities to the subsequent phases of the product life cycle, including disassembly and recycling. This way of thinking about life cycles of artefacts is clearly inspired by biological life cycles: The paradigm shift from cradle-to-grave to cradle-to-cradle is based on the idea that sustainable production is also more economic. Consequently, the designers' tasks and the systems to be designed have become very complex. In the past a products were not reused, repaired, redesigned, reproduced, and today the design is taking into account this. This improve sustainability, saves resources, material, energy, and more. However it might be more complex and/or it have to consider additional factors.

\subsection{Design of bio-inspired surfaces using sensors and bio- integrated materials}

The surface structure and texture haves a significant influence on the function and performance of components and machine tools [1, 6 , and 7], and more specifically, on the design of the small mobile machine used here as the demonstrator. Investigation of bioinspired surface structures using sensors and the development of bio-integrated layers are conducted at the Volcani center in Israel. Layers or sensors adapted on components are able to protect surfaces of the machine tools against environmental influences. Active antimicrobial coatings (Figure 1 b, c, d) and superhydrophobic coatings (Figure 2) as well as surface texture (Figure 1a) are of particular interest for designing technical surfaces. Examples include the structure of cats' tongues with horn tooth for drinking water (Figure 1a), or the snakeskin inspiring texture through additive manufacturing for mechanical traction [9]. Figure $1 \mathrm{~b}$ represents a general idea of attaching antimicrobial compounds to the material's surface in order to form contact-active antimicrobial materials. Figure 1c represents the detailed attachment procedure of phenolic linker as an antimicrobial compound, added to the surface of stainless steel components. In Figure 1d, the antimicrobial compound of quaternary ammonium salt is covalently bound to the previously attached phenolic linker, resulting in the contact-active antimicrobial surfaces.
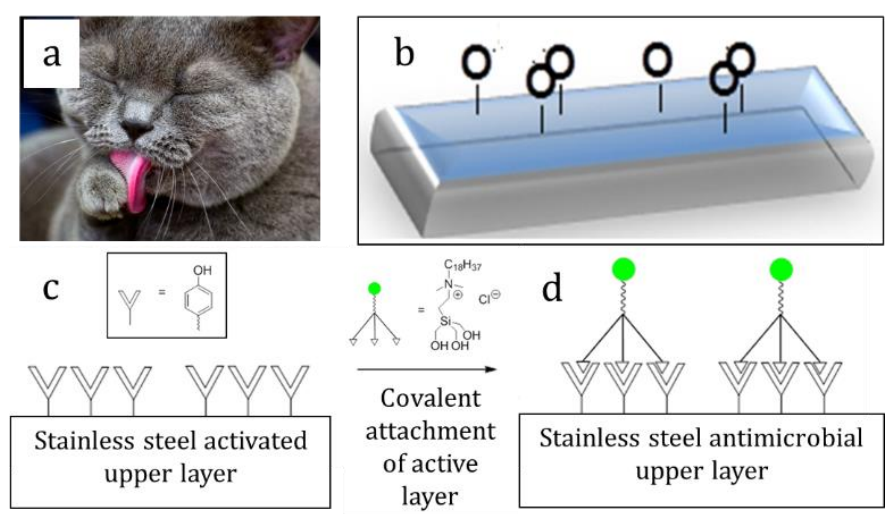

Figure 1: a. An antimicrobial surface b. schematic representation of active nano-coating. c. linker allowing to add active antimicrobials on stainless steel d. antimicrobial nano-coatings.

Durable and transparent super-hydrophobic coatings for plastic, glass and metallic substrates have recently been developed by Mechraz at Volcani center. The new technology for superhydrophobic coatings is based on Pickering emulsions, enabling obtaining a unique combination of nano and micro scale roughness that is required in order to exhibit behavior of superhydrophobicity. Figure 2 shows the waterdroplet motion on polypropylene (a) and polycarbonate (b) sheets without wetting, demonstrating the superhydrophobic behavior. The antimicrobial nano-coatings are strongly (covalently) attached to the surface and are very stable in severe environmental conditions (acidity, temperature, chemical reagents). This antimicrobial 
nano-coating can also protect inner surfaces of machine tool components (such as tubes and hoses) from clogging and damage. Durable and transparent anti-biofilms coatings are also developed $[10,11,12]$.
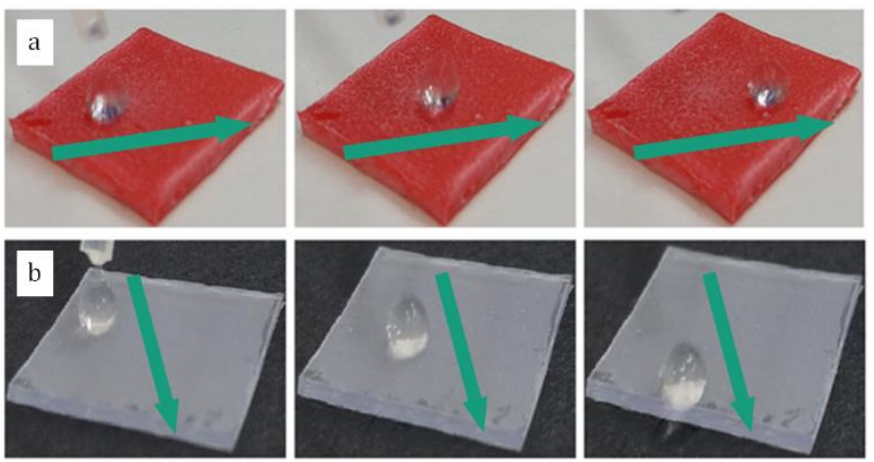

Figure 2: Polypropylene (a) and polycarbonate (b) sheets coated with transparent and durable superhydrophobic coatings developed by Mechraz at Volcani center.

\subsection{Bi-directional inspiration approach to design components, machine tools and systems}

Analyzing bio-based principles can help find a solution to an existing engineering problem (top down), or it can trigger an idea for a new product (bottom up). In the first case the problem specification is the input for a systematic search after suitable biological principles (problem driven, engineering pull), while in the second case the goal is creating a useful implementation of a biological principle for a new product (solution-driven biology push) $[1,13,14,15,16]$. In the latter case, the goal is turning observations of biological principles into equivalents that can be implemented in the artificial world.

R\&D activities at the Mechanical Engineering Department of the Braude College, Israel, together with the School of Zoology in Tel Aviv University, were tailored towards the goals of the current investigation. The activities are based on defined design requirements as well as on biological features and principles, included ongoing research and development to provide novel bioinspired insights, mainly by studying and analyzing insect locomotion as a model for miniature (yet scalable), for optimized structures and for adaptable, dynamic robotics. Using the results of the growing range of available biological data, the up-to-date knowledge, IT and new technologies made it possible to develop new design ideas, new products and new manufacturing systems.

The design and investigation of the cylindrical and toroidal pressure vessel, carried out by [15] and shown in Figure 3a, is aiming to reduce its weight while maintaining the load carrying capacity. This could be obtained through geometrical changes based on FEA, using AM technologies and inspiration or implementation of biological principles (top down approach). The optimization procedure, which started from a vessel similar to a "peanut" shape, using finite element stress analysis, resulted in weight savings of $26 \%$ compared to a domed cylindrical counterpart, and $63 \%$ for an asymmetrical torus compared to the symmetrical baseline, both while keeping the internal volume and pressure, and the allowable stresses. The non-mathematical optimization procedure implementing an original stronger 'peanut" shape can replace cylindrical pressure vessels in weightsensitive applications such as fuel cells in the automotive and aerospace industries. In the production of prototypes and small quantities, conventional manufacturing can be replaced by more efficient additively manufactured parts. a. Design for weight reduction and higher strength of pressure vessels (top down)
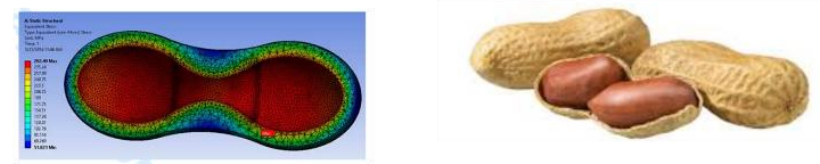

b. Learning from biology to design components and systems (bottom up) Locust - inspired design of a jumping robot Grasshopper, desert locust

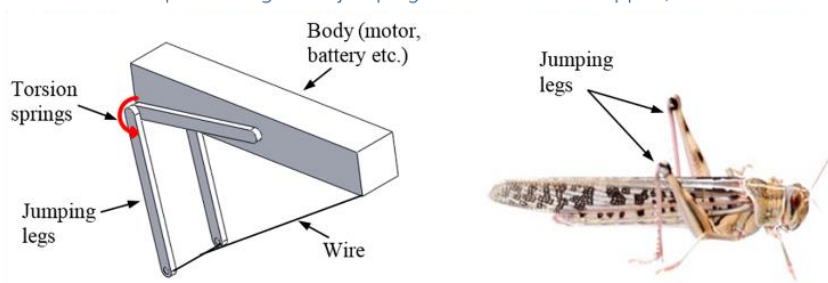

Figure 3: Conceptual design of biologically inspired components and systems $[15,16,17,18,19]$.

The development of the jumping robot, which is shown in Figure $3 \mathrm{~b}$ and designated TAUB, is based on zoology knowledge captured by Ayali from the Tel Aviv University $[17,18,19]$ and investigated by Ben-Hanan at the Ort-Braude College [16]. Among different motion gaits, jumping is a quick and efficient locomotion for advancing in rough, uneven or unpredicted terrains, used by a variety of animals. The observed and applied biological phenomenon and dynamic principles can be considered as bioinspiration but also as bio-intelligence. As the muscle power generation is proportional to its cross-sectional area, small-scale jumpers suffer from power limitation in their tiny muscles. To overcome this barrier, nature created special mechanisms inside these jumpers, enabling them to be propelled for very impressive ranges with respect to their own size. The governing principle comprises loading energy into elastic elements, and at the right time, releasing it as quickly as possible. Researchers designing miniature artificial jumping mechanisms for improving the mobility of small-scale robots face similar power limitations of motors and batteries. The very same principles used by small-scale jumpers are implemented in the bio-inspired artificial mechanisms, and are utilized in the jumping robot. Inspired by the desert locust, the focus lies on the main bio-mechanical principles of the locust's jump rather than attempting to produce an exact imitation of its body and morphology in order to create a simple yet effective jumping mechanism. The demonstrated mechanism is closer to nature's design and could be considered as "biointelligent".

Mobility investigations by [20] used a 2D simulation, which mimics caterpillar locomotion using Assur tensegrity structures with a set of elements always under compression and a set of elements always under tension. The biological caterpillar can inspire mobility controlled mechanical structures. Together with the suggested control scheme, the unique engineering properties of Assur tensegrity structures provide the model with a controllable degree of softness so that each segment can be either soft or rigid. Based on existing and the bi-directional biologically based design procedures, the emerging technologies of Industry 4.0 (I4.0) such as digitalization, digital twins and the Internet of Things (IoT) enable bio-enhanced design methods, the development of new products and advanced manufacturing systems. 
The biologically based design methodologies for advanced manufacturing

\subsection{Design for sustainability using Artificial Intelligence and AM}

New design methodologies of products, processes, machines and systems combine biological principles with sustainability considerations such as higher resource and energy efficiency, use of lightweight components, integration of multiple functions and social conditions, and respecting environment issues [21, 22]. The development of new technologies such as Additive Manufacturing (AM) and the application of digitization and new computing methods, simulation, Artificial Intelligence (AI) and modeling methods enables the design, development and implementation of up-to-date products as shown, for example, in Figure 4.

An approach to identify the optimal design is the use of the Generative Design method in the CAD software. After defining the desired properties of the components and the essential boundary conditions such as material, mass, maximum loads and cost, the software compiles a multitude of designs using algorithms and logical calculations to analyze the given requirements. Based on these versions the designer refines the specification and restarts the calculation. Using AI leads to completely new designs with reduced development time.

The application of topological optimization and Artificial Intelligence-based design methods for Additive Manufacturing made it possible to optimize the machine gripper finger shown in Figure 4 with benefits to efficiency, material saving, and sustainability. The transformation of biological principles into the design of the gripper demonstrator reduces the weight from 312 grams to only 50 grams. Even in the case of a moderate weight reduction to 132 grams, the embodied energy demand is reduced by $40 \%$ compared to the reference design.

Main advantages of AM comprise the compact, lightweight, and customized design of components and the manufacturing of geometrically complex parts, thus reducing assembling efforts. Optimized geometries are often inspired by bionic structures [23, 24]. Integration of sensors, for example motivated by cat's whiskers, into the additively manufactured structure presented in Figure 4 can upgrade components, machine tools or systems to bio-intelligent or "living" products. Manually inserting the sensor into a pre-fabricated cavity and the subsequent finishing of the component by AM enables the integration of commercially available sensors. A more elegant and automatable strategy could be to print sensors directly into the component during the AM process. However, this is an ongoing research work.

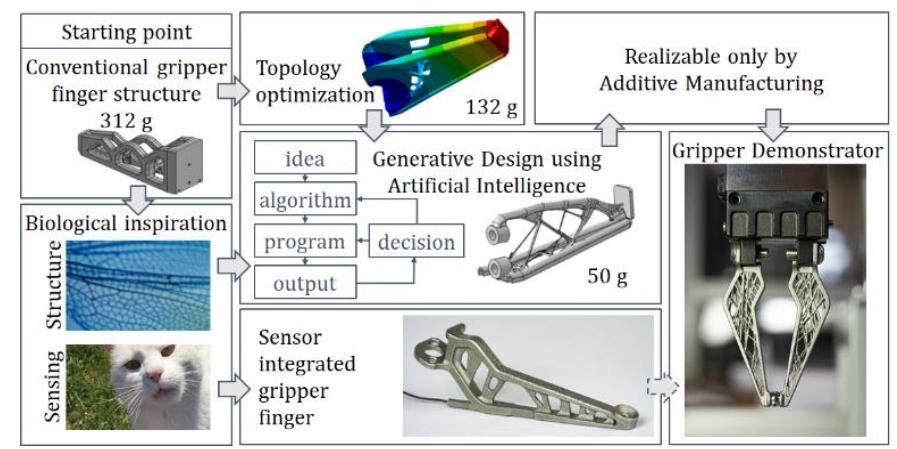

Figure 4: Process of bio-inspired design of additively manufactured lightweight gripper fingers with integrated sensor (in this case strain gauge to monitor gripping pressure).

\subsection{Computational Design Synthesis}

Figure 5 illustrates the evolutionary design approach incorporating the process of synthesis, analysis, evaluation, and adjustment required in CDS - Computational Design Synthesis. This design approach makes the implementation of CDS relatively straightforward.

The research program on Computational Design Synthesis at the University of Twente has been inspired by the idea that the presently available computational power can be used to generate large numbers of design solutions that all adhere to specified constraint sets instead of trying to optimize a single solution to fit a set of requirements. The latter usually induces numerous iterations. Similar to biology, if the resources are abundant, effectivity is a more viable goal than efficiency.

Evolutionary approaches in CDS are based on the principles of biological evolution, and encompass a broad range of search techniques, among which the most prevalent are genetic algorithms, evolutionary programming and evolution strategies [25-31]. The search algorithms of evolutionary approaches are based on genetic adaptive operators, namely, mutation and

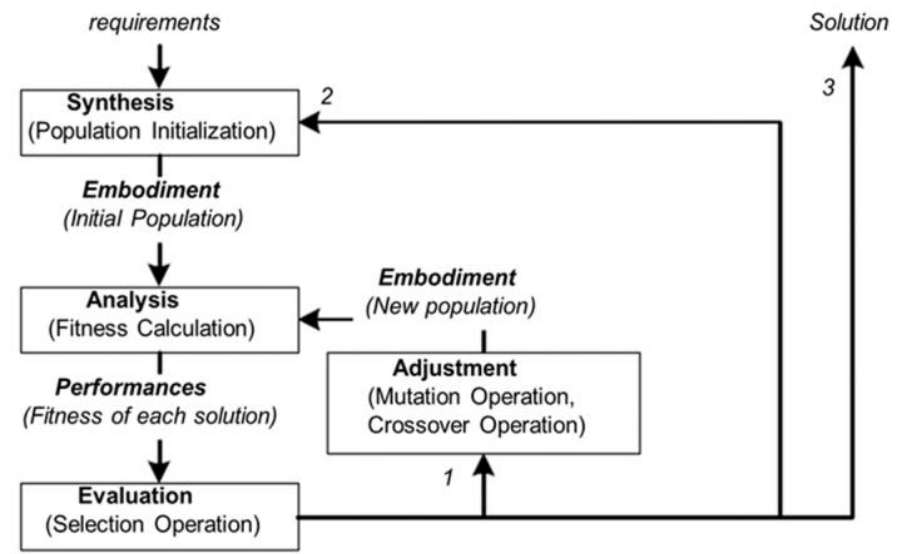

Figure 5: Computational Design Synthesis (CDS), survival of the fittest.

recombination. The selection is based on Darwinian selection, or selection of the fittest. A characteristic of these methods is that they employ sets of solutions (populations) represented by vectors. The structure of the program is fixed with the parameters being the ones allowed to change. Evolutionary programming uses vectors of real numbers for the representation of solutions. The selected solution has the characteristic that the results of mutations are considered in future generations only if they fit better than other solutions found. Evolutionary approaches incorporate the processes of synthesis, analysis, evaluation and adjustment required in $\mathrm{CDS}$ as shown in Figure 5. This characteristic makes the implementations of such approaches relatively straightforward for CDS.

\subsection{Combination of the CDS with ontology based data}

However, it is a labor-intensive procedure to compile these biological principles by hand and to place them in an ontology. By combining CDS with the method of linking engineering and biology, described in chapter 4 , it will become possible to implement bio-inspired design in a more systematic and scalable way. The biologically inspired design cycle presented in Figure 6 is investigated on a mobile machine tool used as a demonstrator during this investigation. The demonstration cycle is described by various functional, bio-inspired surfaces for different applications 
such as tool and die making for micro-injection molding, forming processes and others $[25,26,27,28]$. This may lead to more effective tools for design support. Generating numerous candidate design solutions and reducing the population by specifying additional constraints finally leads to a set of design alternatives that are "equally good". When the set becomes small enough, the remaining solutions can be further elaborated in the classical way by human intervention [25-32].

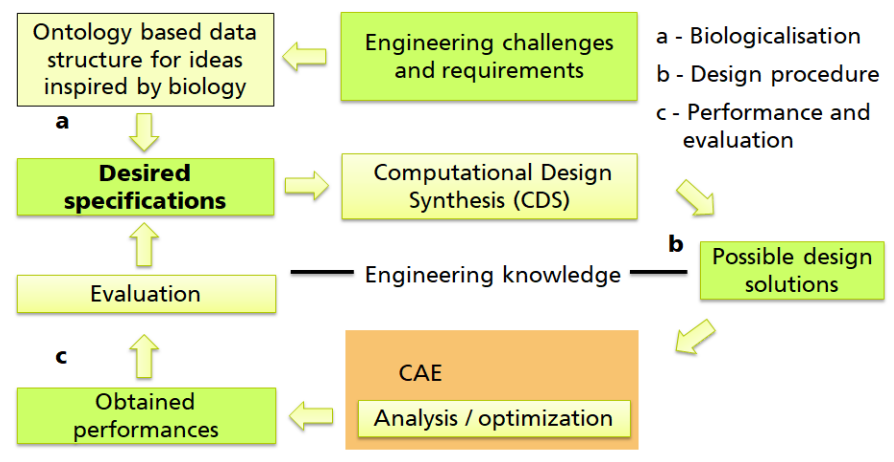

Figure 6: The new design methodology with the biologically inspired design cycle and CDS.

\section{Systematically finding biological principles for a given design problem}

The methodology of biology-based design cycles described in chapter 3 is using relevant biological principles in the design and optimization of new products. Furthermore, this methodology applies new ideas in the manufacturing chain. However, retrieving relevant principles from biology for a given design problem is currently a manual time-consuming step. The following chapters detail the automated systematic search process applied in this investigation. In a future stage, the systematic search method can be combined with CDS to create an end-to-end solution. Chapter 4.2 presents systematic investigation results of biological principles to comply with a given design problem.

\subsection{Systematically matching biological principles to a given problem}

To create bio-inspired designs, engineers need access to relevant biological knowledge during concept generation. Previous research has shown that it is hard to identify a relevant biological strategy for a given design problem and to extract the underlying working principles [33]. Abstracting the working principle requires a deep understanding of the biological strategy before any analogies can be created between biology and engineering. Furthermore, engineers often lack biological training [34], causing most current bio-inspired designs to have relied on a chance observation of a biological phenomenon to obtain the relevant biological principles on which they are built [35]. Another difficulty of using natural language as a source of biological knowledge for engineering is the different vocabulary used in both domains [35]. Recognizing the need to provide a systematic method of accessing bio-inspiration, researchers developed several initiatives, with the Ask Nature database being the most prominent one. However, these initiatives suffer from scalability issues [14]: every biological principle must be manually prepared before it can be added to the database.

A large wealth of biological knowledge is contained in natural language format in biological publications. In [36], the authors proposed a natural language approach that finds and extracts relevant biological strategies based on a functional query. However, the amount of biological knowledge captured is limited as the source material of this proposed search method only comprised a single introductory biology book.

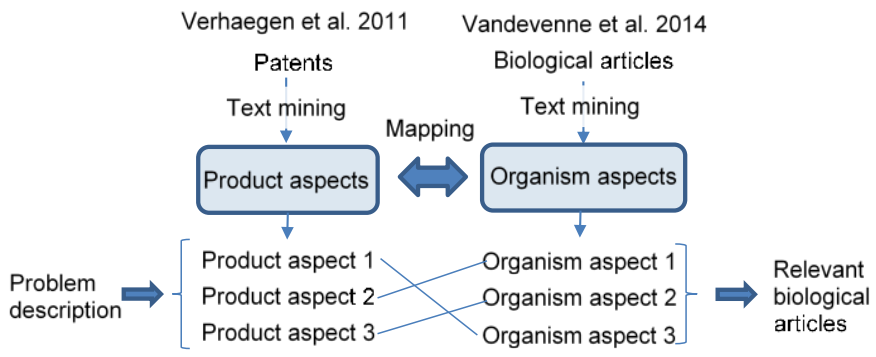

Figure 7: Schematic representation based on the method proposed by Vandevenne [37, 38]. The mapping between product aspects and organism aspects links the problem description with relevant biological articles [14].

Starting from the biological knowledge captured in biological articles, Vandevenne et al. proposed the strategy illustrated in Figure 7 [37, 38]. Latent semantic analysis reveals the set of aspects that are recurring throughout biological literature. These aspects consist of a set of concepts, detected via word cooccurrences in the documents [37]. For example, in the biology literature the organism aspect related to flying might capture vocabulary related to flying and gliding as well as vocabulary related to wings. A very similar approach has been applied to patents, yielding so-called product aspects [38]. These aspects capture the functionality in terms of the vocabulary used in their respective domains. A mapping between the organism aspects in the biology domain and the product aspects links the aspects that deal with similar functions/concepts, taking into account the difference in vocabulary between both domains. This allows bridging the gap between engineering and biology by converting the engineering formulation of the design problem into product aspects and by retrieving the linked organism aspects via the mapping. Finally the organism aspects lead to the most relevant biological publications for the given problem description in the engineering domain.

The current investigation required changing the input method of the engineering problem. By asking the engineer to abstract the problem statement into the essential function that is required, a functional query is formed, which is then translated into corresponding organism aspects. This essential function is an application independent formulation of the required functionality [39].

The natural language method aids in identifying the articles that contain the relevant biological principles. However, the engineer will still have to extract these principles and apply them to the final solution via an analogical transfer. Shu (2010) and Helms et al. (2009) highlight several pitfalls during this process: oversimplification of biological functions, directly copying of the biological strategy due to poor understanding of the underlying principles and fixation on the first encountered strategy $[13,40]$.

The goal of bio-inspired design, in contrast to biomimicry, is not to copy the strategy exactly but to extract the underlying principle and apply this to create a solution in engineering.

During the abstraction of the working principle, a possible influence of the environment has to be taken into account. However, the innovative principle is not bound to the biological 
environment and can be generalized in engineering. For example the shape of the beak of the kingfisher (used in water) is also useful for the difference between the density of the air in and outside of a railway tunnel as successfully applied on the Shinkansen High speed train.

\subsection{Systematic investigation of biological principles to comply with a given design problem}

The new methodologies require systematical investigations of biological principles and biological phenomena to solve problems and to develop products by combining experts in IT, biology and engineering. In spite of great advances in the field of bio-inspired technology, natural systems still retain significant advantages over engineered systems in numerous aspects, including size, and mostly, adaptability and versatility. Insects, in particular, exhibit an unmatched ability to content with a range of perturbations, maintaining control and stability while navigating through a wide variety of environments.

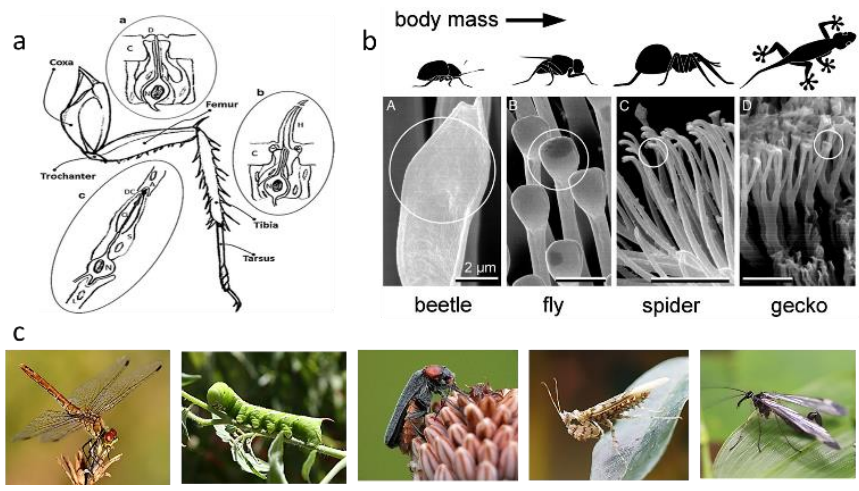

Figure 8: Biological features inspiring engineering solutions a. cockroach sensors [42], b. nanoscale fibrillar structures in animals with varying hair density [41], c. insects with varying body plans for mobility and flexibility.

In several research programs at the school of Zoology of Tel Aviv University the locomotion principles of animals and insects have been studied in order to identify principles that may successfully be applied to engineering problems and to the design of products [17-20, 41-46].

Figure 8 illustrates several unique features of animals and insects, which can inspire investigations and possible implementation in manufacturing. Figure 8a shows a cockroach leg with mechanoreceptors having a role in load sensing and serving as major proprioceptors utilized in different locomotion-related tasks [41, 42]. Examples for Figure $8 \mathrm{~b}$ comprise recent publications in the last two years, describing gecko adhesion phenomena [47], integrative biology of gecko adhesion and biological understanding of the phenomena, [48] as well as the gecko-inspired adhesive materials and application [49]. [50, 51 and 52] present examples for inspiration and optimization from social insect behavior and nature-inspired algorithms for optimization as well as swarm intelligence. These features are used in biologically inspired solutions that can lead to the development of sensors and control systems to be applied in machine tools.

A novel research direction has been initiated, as described in chapter 2, testing the feasibility and functionality of hybrid biotechnology systems. Specifically, testing the utility of biological sensors (i.e. insect sense organs such as ears and antennae) mounted on and incorporated into mobile machines (robots) for the construction of a combined organic-synthetic system. Figure
$8 \mathrm{~b}$ shows the nanoscale fibrillar structures in animals with hairy attachment pads. The density of surface hairs increases with the body weight of animals and can inspire engineering solutions for problems such as clamping, surfaces or adherence, or mobility for better adherence. Figure 8c presents few examples of insects with four and six legs to ensure flexibility, stability and mobility.

However, it is not obvious which configurations and combination of features perform better. In addition, under the theme of biointegration and bio-intelligence, investigations are currently carried out to reveal the rules and algorithms utilized in the generation of collective-coordinated motion in insect swarms. The aim is to apply these in a mixed swarm of insect and miniature mobile robots. Successful incorporation of robots in the biological swarm will open the way to bio-inspired "living" swarm robotics.

5

Parallel kinematic mobile machine tool used as demonstrator for the new design methodology

A parallel kinematic small, mobile and flexible machine tool was selected as the demonstrator $[15,53,54]$. The investigation was carried out according to the new design methodology based on the bio-inspired design cycle described in Figure 6 (chapter 3 and 4). Establishing and analyzing a list of challenges and requirements is discussed in chapter 5.2. Chapter 6 describes ontology based data structure for ideas inspired by biology and analyses of relevant biological principles. Chapter 7 presents conclusions and ideas for the implementation of the biologically inspired design cycle methodology, including the use of Computerized Design Synthesis (CDS), related to structure, mobility, flexibility, clamping possibilities and surface features.

\subsection{Application, structure and features of a mobile machine tool as demonstrator}

The development and investigation on new mobile, flexible and small machine tools is a promising and suitable area to apply these novel insights in the design of surfaces, components, machine tool structures and systems with required functions, mobility and flexibility. Fraunhofer IWU in Chemnitz has ample experience in designing, developing and testing of mobile machine tools, and more specifically regarding parallel kinematic (PK) machines as shown in Figure 9. The research has been conducted on mobile machine tools that are relatively small compared to the workpiece [53-58]. The machine design allows for milling, drilling and similar operations in various directions and positions.

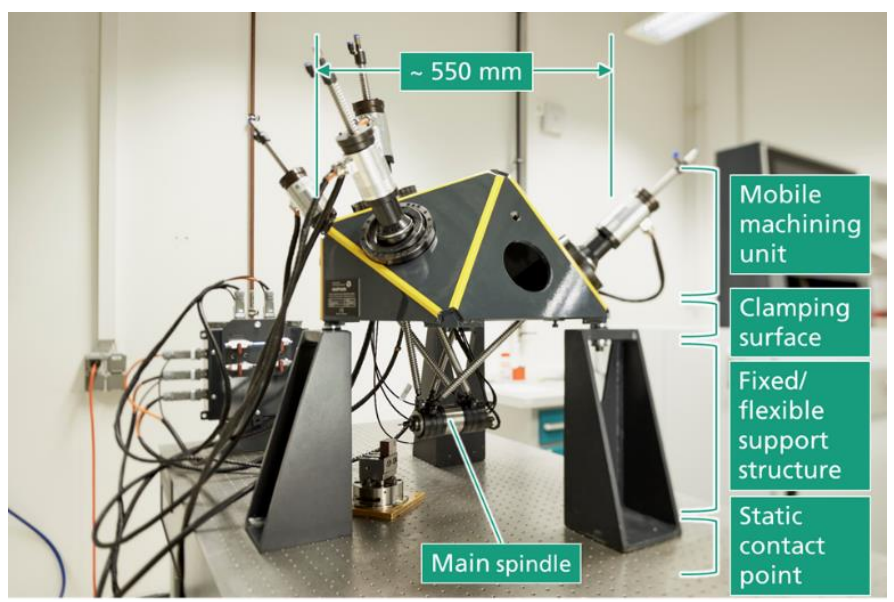

Figure 9: Miniaturized parallel kinematic machine tool at Fraunhofer IWU. 
"Biological small machines ", shown in Figure 10, can be an inspiration for the design of machine tools with different levels of mobility and complexity, ranging from simple manual positioning, to supported structures, to automated enablers (robots) or to autonomous systems. This type of machines and machine components should be able to position, move, clamp and secure themselves with respect to the workpiece and be able to safely execute the manufacturing process within the required accuracy $[15,53-57]$.

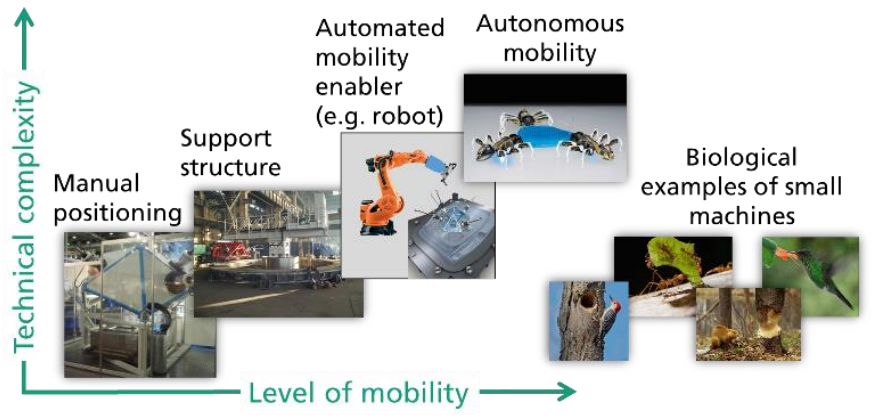

Figure 10: "Biological small machines" and machine tools or systems with different levels of mobility and complexity.

\subsection{Requirements and challenges regarding a parallel kinematic mobile machine}

The analysis of the requirements and challenges for the selected demonstrator was conducted, as an example, on the typical application of the tool and die-making domain, producing large components. The investigation focused on conventional machining processes where mobile solutions can be useful. This is still a wide application field with different materials, part dimensions, and machining processes. The first step is the identification of options for process chain optimization and flexible technology integration in mobile and flexible machine tools. The following variants of flexibilization are imaginable: a machining unit with various adaptable processing heads for flexible technological integration, or a mobile machining unit that can be clamped onto the workpiece for machining within a production line.

In general, small mobile machine tools have to machine large workpieces incrementally. For this reason, re-arrangement of the machine on the workpiece will be necessary and has to be carried out quickly (Figure 11). There are approaches to use support systems (e.g. robots) to automatically position mobile machines on large workpieces. In this case, manual interfaces would not be practical. The additional function would be to enable the machine tool to move to and to clamp autonomously onto the workpiece.

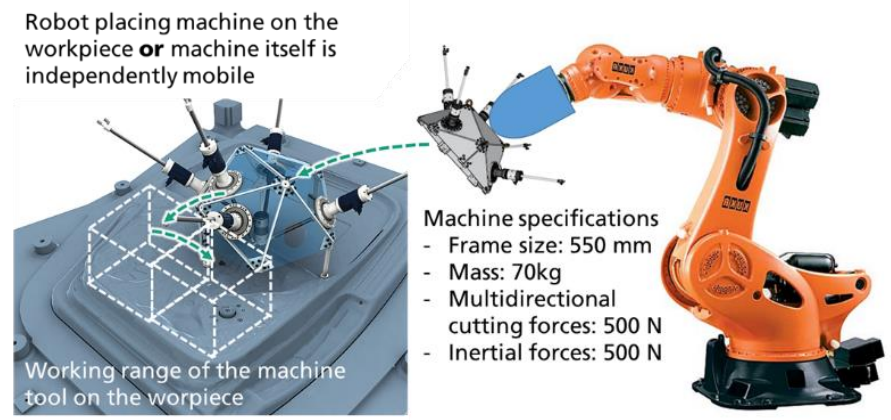

Figure 11: Features, requirements and challenges regarding the mobile machine tool.
The machines must be able to move or be moved over a surface up to an accurately defined position, clamp themselves to the surface and deliver the required stiffness. The surfaces may have quite different properties in terms of materials, shapes and roughness. Sustainability aspects, including saving of resources and energy, environmental issues and social considerations are included in the desired specifications. Although the basic design principles are known, the most promising configurations must be investigated. By using CDS, a population of candidate solutions can be generated and systematically reduced by specifying additional constraints.

Figure 12 presents a list of features, requirements and challenges for the mobile machine tool to be investigated with the new design methodology, including the bio-inspired CDS. The features indicated in green are analyzed and discussed in this paper.

\begin{tabular}{|l|}
\hline Challenges and requirements \\
- Variable mounting options for mobile solutions \\
- Machine structures adapted to size and material \\
- Smart self-calibrating system \\
- Environment protection and sustainability \\
- Lightweight \\
- High stiffness \\
- High acceleration and speed \\
- High accuracy and repeatability \\
- High damping behavior \\
- Optimized thermal behavior \\
- Adapted structures and dimensions \\
- High efficiency and effectiveness \\
- Controlling consumption of power/energy and \\
forces/friction \\
- Flexible technology change (e.g. cutting, \\
ablation, handling processes ....) \\
\hline
\end{tabular}

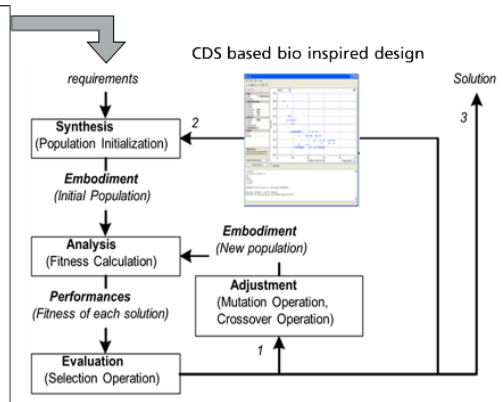

Figure 12: Challenges and requirements for mobile machines.

The following main issues have to be addressed depending on the level of mobility, flexibility, durability and sustainability:

- Intelligent kinematic structures

- $\quad$ Adaptive interface devices enabling machines to adhere to or to be clamped onto various workpiece surfaces and geometries

- Miniaturization and lightweight design to ensure dynamic movement of the machine, even in case of fragile structures

- Smart self-calibration and self-orientation to navigate within the work environment

- Self-awareness in terms of system capabilities in a changing work environment

Such a machine must attach and secure itself to the workpiece and move to spots where operation is needed. While accuracy traditionally comes from the rigidity of the machine frame, movable machines need to obtain their accuracy in another way, depending on the type of manufacturing process.

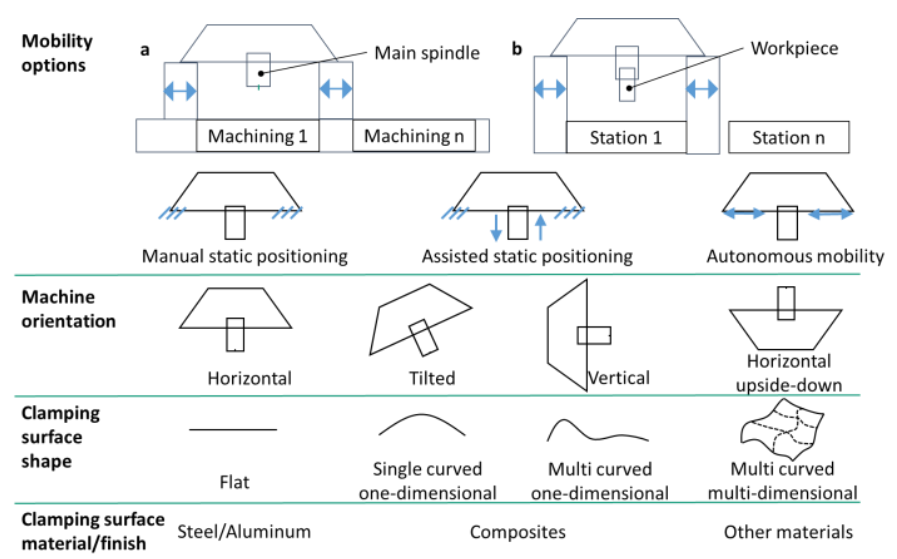

Figure 13: Constraints for the mounting and clamping conditions. 
One of the main purposes of the demonstrator was to find solutions for positioning and clamping, or mounting of those devices. The clamping interface must be very adaptable towards different conditions, but it must provide high stiffness when secured (Figure 13).

- Machine orientation: horizontal and tilted are top priorities; vertical and upside-down are also relevant (e.g. on aircraft structures this application implies higher requirements/challenges due to the machine weight).

- Clamping surface shape: all shapes are possible and important; shapes can differ between different clamping positions.

- Material: steel/aluminum and composites are most relevant.

- Mobile machine function: using clamped workpiece with fixed machining station in a production line or mobile machine with adaptable processing heads.

\section{Investigation of biological data and biological principles for the mobile machine tool}

Following the requirements and challenges presented in chapter 5 , a systematic search was carried out regarding biological data, and an investigation of biological principles was performed. Chapter 6.1 presents the biological strategies and principles retrieved by KU Leuven using the natural language approach detailed in chapter 4.1 to search for relevant bio-inspiration for a clamping interface for the mobile machining unit. Chapter 6.2 includes some relevant results of biological principles and phenomena investigated at the Tel Aviv University. The work is a top down searching procedure based on ontology based data structure looking for ideas inspired by biology, and for the biological research of relevant principles discussed in chapter 4 .

To create a bio-based design of the mobile machine, engineers are using the biological knowledge, presented as an example for clamping and positioning in chapters 6.1 and 6.2 , as a part of the new design methodology shown in chapter 3.3 and in Figure 6.

\subsection{Search results of the systematic bio-inspiration search for the clamping interface between machine tool and workpiece}

Using the natural language approach detailed in chapter 4.1 , a systematic search was performed for bio-inspiration for the clamping interface between the mobile machine tool and the workpiece. The starting point of this search is the essential function, which has to be abstracted from the detailed problem statement described in chapter 5 . Abstracting the design problem leads to the required method of 'reversible attachment', possibly to a 'slippery surface'. This functional query has been used in the natural language system to retrieve a ranked list of biological articles.

The retrieved articles were interactively processed to extract the biological strategies that can be applied to the current investigation. After a quick analysis by a mechanical engineer without a formal biology background, it was noted that the most prevalent species were geckos and ants. Biological strategies present comprise suction pads, used for example by remora fish (e.g. [59]), wet (e.g. [60]) and dry adhesive pads (e.g. [61, 62]) and a combination of these pads with mechanical claws (e.g. [63]). All these examples can be used as bio-inspiration for designing mounting, adhesion and clamping solutions.
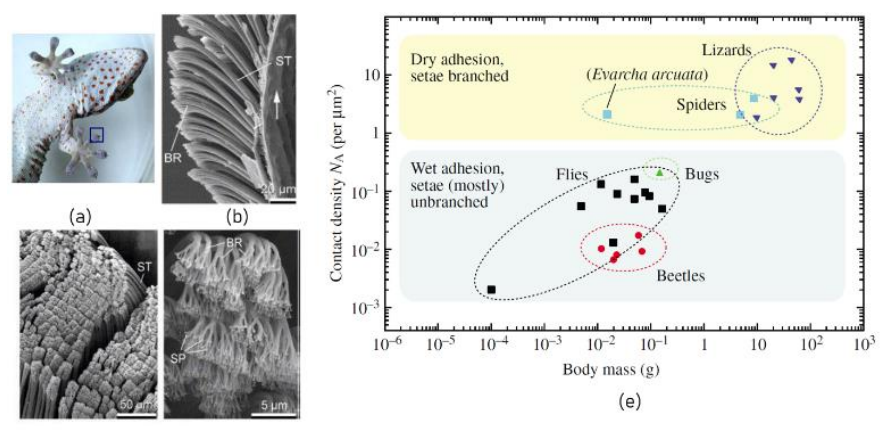

(c)

(d)

Figure 14: Hierarchical adhesive structures of geckos, (a-d) [62] and relationship between contact density $\left(\mathrm{N}_{\mathrm{A}}\right)$ of hairy pads and body mass for animals from a variety of taxa (e) [61].

Figure 14 contains a sample of the images and a summary of their captions that were manually extracted from the relevant papers about the biological strategy that uses adhesive dry pads to provide reversible attachment. In [62], the authors explain how geckos use a hierarchical adhesive system with each branch becoming finer, allowing them to use the van der Waals interaction for attachment (Figure $14 \mathrm{a}, \mathrm{b}, \mathrm{c}$ ). In [61], the authors investigated the relationship between the contact density of the finest points of the adhesion systems of different organisms using different types of adhesive pads (Figure $14 \mathrm{~d}$ ). This figure stems from an identified reviewed paper of the relevant literature, which is especially interesting for designers performing a bio-inspiration exercise since it facilitates comparison of different strategies. This sample serves as an ambassador for the potential of knowledge that is contained in biological articles.

\subsection{Systematic investigation of biological principles related to the mobile machine}

The biological investigations at Tel Aviv University were based on the challenges and requirements of the mobile machine tool as defined in chapters 5.1 and 5.2. As shown schematically in Figure 15, the ongoing bio-inspired research is investigating biological organisms, animals and mainly insects. It was aimed at providing novel bio-inspired insights and principles related to the structure, stability and mobility, clamping, adhesion and mounting, as well as sensors and control systems of mobile machine tools.

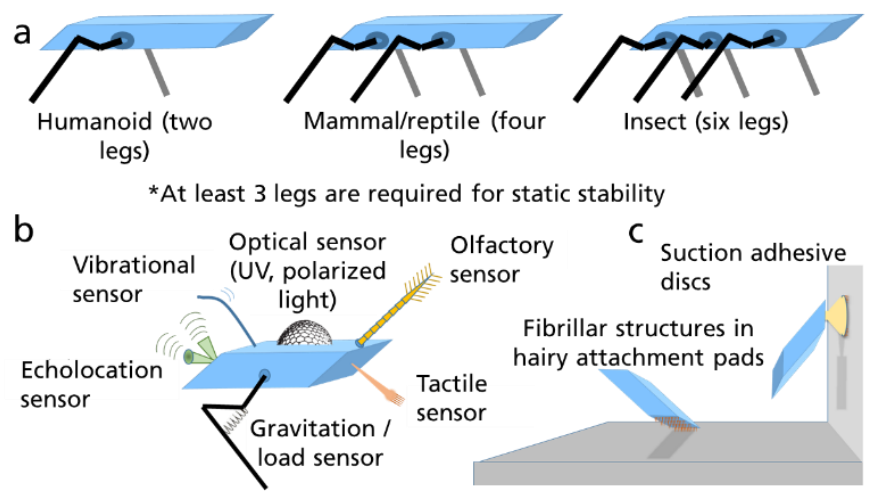

Figure 15: Insect-based bio-inspiration for the mobile machine tool, a) stability and mobility, b) sensors and control system, c) mounting and adhesion.

In particular, this investigation comprised legged machines (Figure $15 \mathrm{a}$ ), biological sensors (Figure $15 \mathrm{~b}$ ), and possibilities for mounting or adhering on the workpiece (Figure 15c). Insect 
locomotion and the relative advantages of feedforward vs. feedback-based control were studied as a model for miniature (yet scalable), adaptable dynamic robotics. In addition, the initiated novel research directions included studies concerning feasibility and functionality of hybrid bio-technology systems for biological sensors mounted on and incorporated in mobile machines (robots) for the construction of combined organic-synthetic systems (Figure $15 \mathrm{c}$ ).

Bio-inspiration from various insects and animals as biological models for mounting and adhesion of is mentioned in chapter 4.2 (Figure 8) and in chapter 6.1 (Figure 14). The nanoscale fibrillar structures with varying hair density and the hierarchical adhesive structure of geckos allow them to move fast and safely on any surface. Their toes contains hundreds of thousands of setae (ST), and each branch (BR) of seta contains hundreds of spatulae (SP), as shown in Figure 14 a. The scanning electron micrographs shows rows of setae at different magnifications (Figure $14 \mathrm{~b}$ ) and the spatulae with the finest terminal branches of setae (Figure $14 \mathrm{c}$ ).

The biological suction function of the northern clingfish, Gobiesox maeandricus, as shown in Figure 16, can be implemented for higher loads and mounting forces [64]. The fish is able to adhere equally well to slippery, fouled and irregular surfaces in the marine intertidal environment, with a broad range of surface roughness from $\mathrm{Ra}=15 \mu \mathrm{m}$, corresponding to finishing textures up to a rough surface with $\mathrm{Ra}=269 \mu \mathrm{m}$. The fish outperforms fabricated suction cups, which only adhere to the smoothest surfaces. The adhesive forces of clingfish correspond to pressures of 0.2-0.5 atm below ambient temperature and correspond to 80-230 times the body weight of the fish. The tenacity appears to be related to hierarchically structured microvilli around the edges of the adhesive disk, which are similar in size and aspect ratio to the setae found on the feet of geckoes, spiders and other insects (Figure 14). This very interesting feature points towards a possible bioinspired solution to the problem of mounting and reversibly adhering to irregular, submerged surfaces of mechanical components.

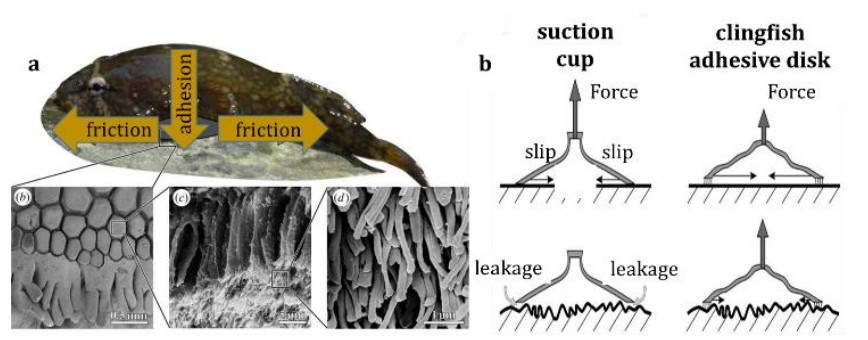

Figure 16: The clingfish with natural suction cups providing high adhesive forces [64].

The systematic biological search (top down) related to mobile machine tools was carried out using the defined requirements, challenges and features looking for bio-inspiration regarding mobility, positioning, adhesion and clamping. Designers can apply these results during concept generation mainly as bio-inspiration, but also for bio-integration and for bio-intelligence of the manufacturing system.

\section{Conclusions and recommendations for the} implementation of the new design methodologies

The joint work showed that bio-inspiration can serve designers in two distinct ways: as a trigger for searching and implementing a solution for an engineering problem (top down), or as a trigger by an observation in the bio-space to be converted or adapted to the technical space (bottom up).

The virtual systematic design method was proved; however, an operational design cycle cannot be demonstrated yet. This paper presents examples for using the new design methodology for different machine elements while fulfilling requirements and challenges. Chapters 7.1, 7.2, 7.3 \& 7.4 present possibilities of implementing the new biology-based design methodology in general, and provide more details for the implementation in the design of a small mobile parallel kinematic machine tool as a typical demonstrator.

An approach and data structure were defined for systematic collection and storage/retrieval of bio-inspired design principles as an input for set-based design.

The collaboration between engineers, biologists and ICT teams resulted in valuable conclusions, recommendations and real applications. The combination and integration of biologically based principles with engineering topics carried out by the different institutes in Leuven (Belgium), Twente, (Netherlands), Chemnitz,(Germany), Tel Aviv and the Volcani Biological Institute (Israel), contributed to the multi-disciplinary design of future efficient and sustainable manufacturing systems.

Investigations regarding the viability of set-based design approach were carried out as an example for the indicated applications. Development of real-time simulation tools and their subsequent investigation can be used in a set-based design platform.

From the biological investigations, it could be concluded that legged locomotion (bio-inspired) is the most advantageous means of locomotion. Six-legged locomotion excels especially on uneven, unexpected terrain and surfaces. Nature provides innumerable examples for the development of sophisticated sensors in a large variety of modalities. Superb sensory-motor perception and decision-making is also ubiquitous in natural systems. Bioinspired principles can serve in devising solutions for mounting and adhesion. Different mechanisms can be selected in a weightdependent and surface-dependent context. Insects can serve as good inspiration for innovation related to miniaturization, stiffness and lightweight.

The numerous biological examples presented in this paper show how the design of products, machine tools, and systems can be bioinspired. Possible design directions are demonstrated and solutions are suggested for a selected demonstrator, dealing with problems such as machine structures and mobility, surface protection, and adaptive clamping devices. However, it is not obvious which configurations perform better, thus actual testing is required.

The benefits of bio-inspiration in manufacturing can be substantially increased by applying systematic mapping of scientific publications of the two domains- the biological-, and the technical one.

The biological principle of effectiveness in achieving goals by means of redundancy can be mimicked by applying methods of Computational Design Synthesis enabled by (massive) digitalization.

The combination of new technologies such as Additive Manufacturing and bio-inspired shape optimization enables lighter, functionally optimized/integrated parts.

The basic biological approach for designing is bio-inspiration, (biomimicry). However, applications such as the use of biological protecting layers and biological fluids are primarily considered as bio-integration topics. The design and use of active sensors, 
Artificial Intelligence (AI) and machine learning (ML) methods can be considered as bio-intelligent processes, products, machine tools, and automated or autonomous "living" manufacturing systems.

\subsection{Using the new methodology to design biology-based products, machine tools, and manufacturing systems}

The results, conclusions and ideas were very useful for implementing the methodology, including the use of the Computerized Design Synthesis (CDS) for future development of the mobile machine. They provided new ideas for the design of mobility, flexibility, stability, strength and dimensions of components such as clamping and mounting devices, machine elements, and structures or surface modifications of products, machines, and systems. Fraunhofer IWU Chemnitz has experience in designing, manufacturing and rating of microstructured and functionalized surfaces for various applications as shown in Figure $17[21,22,65,66,67]$.

Figure 17 also shows some examples of using the new design methodology, described in Figure 6, for different elements of mobile machine tools, fulfilling the requirements and challenges described in chapter 5 .

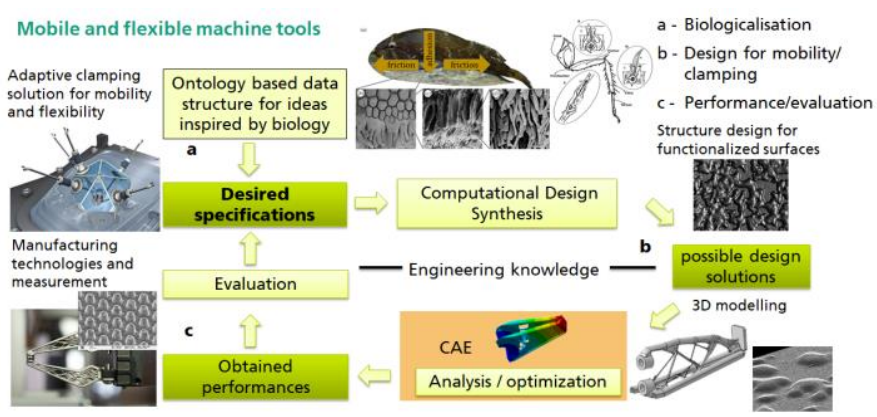

Figure 17: The new methodology to design mobile machine tools including examples of optimized component structures and functionalized surfaces.

For instance, observation of insects' features and behavior (Figure 15 ) leads to the conclusion to investigate mobile machine tools with six legs enabling improved mobility, stability, and flexibility. The idea has already been implemented in mobile robots and moon vehicles. Biological data presented in chapter 3 and 6 regarding animal mobility and movement, including adhesion to smooth surfaces, for example existing in geckos and insects, provided bio-inspired ideas for possible solutions such as clamping and mounting of the small and mobile machine tool and robots.

Biological phenomena such as the natural suction cups of the clingfish providing high adhesive forces (Figure 15) can inspire the design of mobile robots or mobile machine tools moving to a planned position on rough and complex surfaces to carry out operations such as drilling, milling, deburring, cleaning or finishing.

Design of structures, such as the lightweight gripper, is inspired by biological principles, uses AM technologies, and improves sustainability.

\subsection{Sustainability and environmental requirements}

Sustainability requirements and environmental aspects influence the design of products, processes, components, machine tools and the complete manufacturing chain. By following the bio-inspired paradigm of effectivity by redundancy enabled by digitization in set-based design, it becomes possible to consider sustainability as a major boundary condition (constraint) for the search space from the beginning, while in the classical approach of optimization this is usually one of the last items to address.

The UN defined 17 sustainable interconnected development goals also influencing the manufacturing world. Some of these goals such as health and well-being, industry, innovation and infrastructure, responsible consumption of resources and production, and environmental issues have substantial impact on the structural design, use of resources, wear and maintainability of machine tools.

Using structured surfaces and protecting coatings on machine parts, reducing component weight by AM, saving energy and reducing pollution are only few examples discussed in this paper. Replacing expensive, rare and hazardous materials during the design process can fulfil the demand for better sustainability.

\subsection{Bio-integrated and bio-inspired surface modification}

Chapter 2.1 described some biological examples of surface modifications that can inspire the design process. In addition, the use of bio-integrated layer solutions was also described. Machine tools used under severe environmental conditions can be protected by effective biological layers, which can prevent pollution, corrosion, excessive wear and damage. In addition to the sustainability problems, biological and chemical surface treatments can protect the machine against any type of fluids, including new microbial-based fluids.

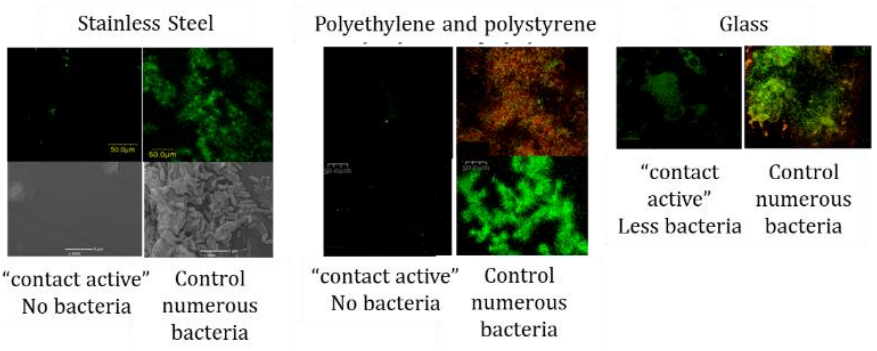

Figure 18: Contact-active coatings - results on various materials.

The Volcani Institute has contributed numerous examples and ample evidence proving that nano-bio coatings can protect various material surfaces. Figure 18 illustrates the surfaces of preliminary tests of the bio-integrated active nano-coating on stainless steel, plastic materials and glass.

\subsection{Bio-intelligent and "living" manufacturing systems}

The proposed definition in the paper "Towards High Performance Living Manufacturing Systems - A new Convergence between Biology and Engineering" by Byrne et.al., published in this same JMST issue, states that "bio-intelligence manufacturing is realized through merging artificial intelligence with bio-inspired and/or biointegrated manufacturing solutions, incorporating information channels, sensors and actuator systems. A special form of biointelligent manufacturing occurs when co-existence, mutual interactions and co-evolution of technical, informational and biological elements take place, with the potential of converging towards living systems".

Will it be correct to define the new design methodology and the resulted developments, based on this definition, as biologically living and intelligent systems?

In chapter 3.1 and Figure 4, the use of Artificial Intelligence (AI) during the design process provided optimal and lightweight 
shapes of components. Today in many other cases AI, ML, DL and biological transformations are used. Chapter 2.1 and Figure 1 illustrated sensors integrated into the coating, which can provide "on-line" information on performance and environmental conditions. In up-to-date machine tools and production systems sensors are used to control "on-line" numerous parameters such as temperature, movements, deformations and other parameters.

The design and use of sensors, control systems, AI and ML can be classified as bio-intelligent processes or as "living" products, processes, machines or systems.

The large amount and versatile types of sensors identified in insects and other organisms could lead to the next generation of bio-inspired or bio-intelligent products, machines and systems. The biological sensors on a cockroach for various functions (chapter 4.2) and the discussed options in Figure 15 can be used for future design procedures according to the new methodology.

Will it be possible to use biological sensors as bio-intelligent elements in manufacturing? Can AI, ML and sensors in products, machines, components and manufacturing systems using similar "living and learning features", be considered to be bio-intelligent?

\subsection{Recommendations and future activities}

Recommendations for future research activities include:

- Extending topological optimization with (embedded) actuated structural elements that can change part/system properties in real time.

- $\quad$ Studying the interaction between microbial cutting fluids and anti-microbial nano-coatings to protect machine elements from pollution and wear.

- Further extending the potential of bio-inspired computational design synthesis.

- Initiating follow-up projects or programs using a working demonstrator including physical and virtual functions.

It is recommended to investigate structural design of parts produced by Additive Manufacturing respecting sustainability and life-like behavior using artificial muscles, sensors and nerves.

\section{Summary}

This paper presents results of an investigation dealing with design methodologies using biological phenomena and data for the development and application of products, machine tools, processes and manufacturing systems. The main biological approach for designing is bio-inspiration. However, links and examples implementing bio-integration and bio-intelligence have also been considered and analyzed during the investigation.

This work describes a systematical design procedure mainly based on a top down approach, starting from requirements and applications related to manufacturing, using well-known theories and experience in engineering. In addition, it is emphasizing and applying biological principles, materials, properties, structures, phenomena and learning from nature.

The growing need to include ever more sustainability issues in design increases the complexity of the design process and that of its outcome (the designed item). Fortunately, the immense and ever-growing capacity of ICT and digitization possibilities provide inspiring options for new, more effective design methodologies. Nature and biological domains can be integrated, can inspire and can be implemented or even applied as intelligent systems in the design procedure.

\section{$9 \quad$ Acknowledgement}

The authors wish to thank the Fraunhofer Gesellschaft and Professor Reimund Neugebauer, President of the Fraunhofer Gesellschaft for the initiation and proactive support with this project. We also acknowledge the support of the Fraunhofer Team at the Think-Tank under the leadership of Dr.-Ing. Sophie Hippmann and Ms. Kerstin Funck (Fraunhofer).

The authors thank the German Federal Ministry of Education and Research (BMBF) for their support in the research project m2FLEX "Miniaturized parallel kinematic production systems for the flexible machining of precision components".

\section{References}

[1] Byrne, G., Dimitrov, D., Monostori, L., Teti, R., v. Houten, F., Wertheim, R. "Biologicalisation: Biological transformation in manufacturing", CIRP Journal of Manufacturing Science and Technology 21 (2018)1-32

[2] Tomiyama, T., Gu, P., Jin, Y., Lutters, D., Kind, Ch., Kimura, F., Design Methodologies and Educational Applications, Annals of the CIRP Vol. 58/2 (2009),

[3] Unglert. J. M. Designing future factories, A novel approach to configuring production systems by combining set-based and automated design. PhD thesis University of Twente, 2018.

[4] Neugebauer, R., Biologische Transformation, Spriger Vieweg, 2019, ISBN 978-3-662-58242-8, https;//doi.org/10/1007/978-3-662-58243-5

[5] Shu, L.H., K. Ueda, I. Chiu, and H. Cheong. 2011. "Biologically Inspired Design." CIRP Annals 60 (2): 673-93. https://doi.org/10.1016/j.cirp.2011.06.001.

[6] Malshe A, Rajurkar K, Samant A, Hansen HN, Bapat S, Jiang W. (2013) Bio-inspired Functional surfaces for Advanced Applications, CIRP Annals- Manufacturing Technology 62 : 607-628

[7] Malshe A, Bapat S, Rajurkar K, Haitjema H,(2018) Bioinspired textures for functional applications, CIRP AnnalsManufacturing Technology 67: 627-650

[8] Jin Y, Zouein G.E., Lu S.C-Y (2009),A Synthetic DNA Based Approach to Design of Adaptive Systems CIRP AnnalsManufacturing Technology 58: 153-156

[9] Bapat S, Tiner C, Rajurkar P, Nath S.D, Atre S. V, Malshe A, (2020) Understanding biologicalisation of the snake-skin inspired textures through additive manufacturing for mechanical traction CIRP Annals- Manufacturing Technology 69/1: 201-204

[10] Elena Poverenov, Moshe Shemesh, Antonino Gulino, Domenico A. Cristaldi, Varda Zakin, Tatiana Yefremov, Rina Granit "Durable contact active antimicrobial materials formed by a one-step covalent modification of polyvinyl alcohol, cellulose and glass surfaces" Colloids and Surfaces B: Bio interfaces 112 (2013) 356-361

[11] Eugene Khaskin, Tania Fadida, Yulia Kroupitski, Moshe Shemesh, Domenico A. Cristaldi, Antonino Gulino and Elena Poverenov "A contact active bactericidal stainless steel via a sustainable process utilizing electrodeposition and covalent attachment in water" Green Chem., 2015, 17, 2344-2347

[12] Tania Fadidaa ,b, Yulia Kroupitskia,b, Uri M. Peiperc, Tatyana Bendikovd,Shlomo Sela (Saldinger)a, Elena Poverenova,*Air-ozonolysis to generate contact active antimicrobial surfaces:Activation of polyethylene and polystyrene followed by covalent graft of quaternary 
ammonium salts. Colloids and Surfaces B: Biointerfaces 122 (2014) 294-300

[13] Helms, Michael, Swaroop S. Vattam, and Ashok K. Goel. 2009. "Biologically Inspired Design: Process and Products." Design Studies 30 (5): 606-22.

https:/doi.org/10.1016/j.destud.2009.04.003.

[14] Vandervenne, Dennis. 2014. "Methods and Algorithms for Systematic Biologically Inspired Design." PHD Dissertation, KU Leuven.

[15] Kroll, E., Buchris, E. (2018). "Weight reduction of 3D-printed cylindrical and toroidal pressure vessels through shape modification." Procedia Manufacturing 21: 133-140.

[16] Ben Hanan, U., Weiss, A., Zaitsev, V., Jumping efficiency of small creatures and its applicability in robotics, Procedia Manufacturing,15th Global Conference on Sustainable Manufacturing, GCSM, (2017)

[17] Zaitsev, V., Gvirsman, O., Hanan, U. B., Weiss, A., Ayali, A., \& Kosa, G. (2015). "A locust-inspired miniature jumping robot." Bioinspiration \& biomimetics, 10(6), 066012.

[18] Gvirsman O., Kosa, G., \& Ayali, A. (2016). “Dynamics and stability of directional jumps in the desert locust. "PeerJ, 4, e2481.

[19] Beck, A., Zaitsev, V., Hanan, U. B., Kosa, G., Ayali, A., \& Weiss, A. (2017). "Jump stabilization and landing control by wingspreading of a locust-inspired jumper". Bioinspiration \& biomimetics, 12(6), 066006.

[20] Orki, Ayali A., et al. (2012) "Caterpillar locomotion model based on Assur Tensegrity Structures." Bioinspiration \& Biomimetics, 7(4): 046006

[21] Drossel, W., Dani, I., Wertheim, R., Biological transformation and technologies used for manufacturing of multifunctional metal-based parts , Procedia Manufacturing, GCSM - 16th Global Conference on Sustainable Manufacturing 33 (2019) 115-122.

[22] Cohen, Y. H., Reich, Y., GREENBERG, S. Sustainability Strategies in Nature, Int. J. of Design \& Nature and Ecodynamics. Vol. 9, No. 4 (2014) 285-295

[23] Neugebauer, R., "Parallelkinematische Maschinen, Entwurf, Konstruktion, Anwendung", Springer Verlag, 2010

[24] Yang, Y., Song, X,. Li, X., Chen, Z., Zhou, C., Zhou, Q., Chen, Y. Recent Progress in Biomimetic Additive Manufacturing Technology: From Materials to Functional Structures, Adv. Mater. 2018, 1706539 https://doi: 10.1002/adma.201706539

[25] J. M. Jauregui-Becker, H. Tragter, and F.J.A.M. van Houten. Structuring and modeling routine design problems for computational synthesis development. In CIRP Design Conference on Design Synthesis 2008, 2008.

[26] J. M. Jauregui-Becker, W. W. Wits, and F.J.A.M van Houten. Reducing design complexity of multidisciplinary domain integrated products: a case study. In 41st CIRP Conference on Manufacturing Systems, volume 1, pages 149-154, 2008.

[27] J. M. Jauregui-Becker, From how much to how many, Managing complexity in routine design automation, $\mathrm{PhD}$ thesis University of Twente, 2010

[28] W. O. Schotborgh, F. G. M. Kokkeler, H. Tragter, M. J. Bommhoff, and Fjam van Houten. A generic synthesis algorithm for well-defined parametric design. Proceedings of the 18th CIRP Design Conference, 2008.

[29] W. O. Schotborgh, F. G. M. Kokkeler, H. Tragter, and Fjam van Houten. A bottom-up approach for automated synthesis tools in the engineering design process. Proceedings of International Design Conference 2006, pp. 349-356, 2006.

[30] W. O. Schotborgh, H. Tragter, F. G. M. Kokkeler, Fjam van Houten, and T. Tomiyama. Towards a generic model of smart synthesis tools. Proceedings of the CIRP Design Seminar 2007, 2007.

[31] W. O. Schotborgh, M.H.L. Roring, D.R. Grigoras, H. Tragter, F.G.M. Kokkeler, and F.J.A.M. van Houten. A development methodology for parametric synthesis tools. In ASME IDETC\&CIE, 2007.

[32] W. O. Schotborgh. Knowledge engineering for design automation. PhD thesis University of Twente, 2009.

[33] Fayemi, P.-E., Wanieck, K., Zollfrank, C., Maranzana, N., Aoussat, A., 2017. Biomimetics: process, tools and practice. Bioinspiration \& Biomimetics 12, 011002. https://doi.org/10.1088/1748-3190/12/1/011002

[34] Hooker, Gretchen, and Ethan Smith. 2016. "Ask Nature and the Biomimicry Taxonomy." INSIGHT 19 (1): 46-49. https://doi.org/10.1002/inst.12073.

[35] Nagel, J.K.S., Stone, R.B., McAdams, D.A., 2010. An Engineering-to-Biology Thesaurus for Engineering Design, in: https://doi.org/10.1115/DETC2010-28233

[36] Cheong, H., Shu, L.H., 2014. Retrieving Causally Related Functions From Natural-Language Text for Biomimetic Design. Journal of Mechanical Design 136, 081008. https://doi.org/10.1115/1.4027494

[37] Vandevenne, Dennis, Paul-Armand Verhaegen, Simon Dewulf, and Joost R. Duflou. 2015. "SEABIRD: Scalable Search for Systematic Biologically Inspired Design." Artificial Intelligence for Engineering Design, Analysis and Manufacturing 30 (01): 78-95. https://doi.org/10.1017/S0890060415000177.

[38] Verhaegen, P.-A., J. D’hondt, D. Vandevenne, S. Dewulf, and J.R. Duflou. 2011. "Automatically Characterizing Products through Product Aspects." In Global Product Development, edited by Alain Bernard, 595-605. Berlin, Heidelberg: Springer Berlin Heidelberg. https://doi.org/10.1007/978-3642-15973-2 60.

[39] Pahl, G., Wallace, K., Blessing, L., Pahl, G. (Eds.), 2007. Engineering design: a systematic approach, 3 rd ed. Ed. Springer, London.

[40] Shu, L.H. 2010. "A Natural-Language Approach to Biomimetic Design." Artificial Intelligence for Engineering Design, Analysis and Manufacturing 24 (04): 507-19. https://doi.org/10.1017/S0890060410000363.

[41] Arzt, E., Gorb, S., Spolenak, R., 2003. From micro to nano contacts in biological attachment devices. PNAS 100 10603-10606. https://doi.org/10.1073/pnas.1534701100

[42] Ayali, A., et al. "Sensory feedback in cockroach locomotion: current knowledge and open questions." Journal of Comparative Physiology A 201.9 (2015): 841-850.

[43] Ayali, A., et al. "The comparative investigation of the stick insect and cockroach models in the study of insect locomotion." Current Opinion in Insect Science 12 (2015): 1-10.

[44] Ariel, G., Ayali, A. (2015) "Locust collective motion and its modeling. " PLoS computational biology, 11(12), e1004522

[45] Knebel D., Ayali A., Pfl?ger HJ, and Rillich J. (2017) "Rigidity and flexibility: The central basis of inter-leg coordination in the locust." Frontiers in Neural Circuits 10:112

[46] Reches E., Knebel D., Rillich J., Ayali A. and Barzel B. (2019) "The metastability of the double-tripod gait in locust locomotion." iScience 12, 53-65.

[47] Bhushan, B. (2018). Gecko Adhesion. In Biomimetics (pp. 739-817). Springer, Cham.

[48] Russell, A. P., Stark, A. Y., \& Higham, T. E. (2019). The integrative biology of gecko adhesion: historical review, current understanding, and grand challenges. Integrative and comparative biology, 59(1), 101-116. 
[49] Wang, L., Hui, Y., Fu, C., Wang, Z., Zhang, M., \& Zhang, T. (2020). Recent advances in Gecko-inspired adhesive materials and application. Journal of Adhesion Science and Technology, 1-17

[50] Bonabeau, E., Dorigo, M., \& Theraulaz, G. (2000). Inspiration for optimization from social insect behaviour. Nature, 406(6791), 39-42.

[51] Fister jr, Iztok \& Yang, Xin-She \& Fister, Iztok \& Brest, Janez \& Fister, Dušan. (2013). A Brief Review of Nature-Inspired Algorithms for Optimization. Elektrotehniski Vestnik/Electrotechnical Review. 80.

[52] Chakraborty, A., \& Kar, A. K. (2017). Swarm intelligence: A review of algorithms. In Nature-Inspired Computing and Optimization (pp. 475-494). Springer, Cham.

[53] Georgi, O., Rentzsch, H., Blau, P.: Miniaturized parallel kinematic machine tool for the machining of small workpieces, 18th International Conference of the European Society for Precision Engineering and Nanotechnology, EUSPEN 2018. Proceedings : June 4th-8th June 2018, Venice, IT Bedford: Euspen, 2018 ISBN: 978-0-9957751-2-1 S.177178

[54] Rentzsch, H. , Georgi, O.,: Modular und autark - Maschinen und Komponenten für die agile Produktion, Präzision durch Adaptive Produktion. 10. Chemnitzer Produktionstechnisches Kolloquium, CPK 2018 : 6.-7. Juni 2018, Chemnitz Chemnitz: Fraunhofer IWU, 2018 S.115-119

[55] Rentzsch, H. , ; Witt, S.; Schwenke, H.: Bearbeitung komplexer Großbauteile mit mobilen Werkzeugmaschinen, wt Werkstattstechnik online Jahrgang 109 (2019) H. 1/2

[56] Rentzsch, H.; Kolouch, M.; Putz, M.: Application of substructure techniques to predict cutting stability for mobile machine tools, 16th CIRP Conference on Modelling of Machining Operations, Procedia CIRP 58 ( 2017) 110 - 115

[57] Rentzsch, H.; Ihlenfeldt, S.: Predicting mobile machine tool dynamics by experimental dynamic sub structuring, International Journal of Machine Tools and Manufacture 108 (2016), S.127-134

[58] Harpaz, O., Books, B., Schwaar, M., Schubert, A., Eckert, U.: Parallel high speed machining with a new additional HSC spindle for machine tools, 5th CIRP Conference on High Performance Cutting 2012 : 4.-7. June 2012, Zürich; HPC 2012 Amsterdam: Elsevier, 2012 (Procedia CIRP 1.2012) S.673-674

[59] Beckert, M., Flammang, B.E., Nadler, J.H., 2015. Remora fish suction pad attachment is enhanced by spinule friction. Journal of Experimental Biology 218, 3551-3558. https://doi.org/10.1242/jeb.123893

[60] Scholz, I., Barnes, W.J.P., Smith, J.M., Baumgartner, W., 2009. Ultrastructure and physical properties of an adhesive surface, the toe pad epithelium of the tree frog, Litoria caerulea White. Journal of Experimental Biology 212, 155162. https://doi.org/10.1242/jeb.019232

[61] Federle, W., 2006. Why are so many adhesive pads hairy? Journal of Experimental Biology 209, 2611-2621. https://doi.org/10.1242/jeb.02323

[62] Gao, H., Wang, X., Yao, H., Gorb, S., Arzt, E., 2005. Mechanics of hierarchical adhesion structures of geckos. Mechanics of Materials 37, 275-285. https://doi.org/10.1016/j.mechmat.2004.03.008

[63] Kesel, A.B., 2003. Adhesion measurements on the attachment devices of the jumping spider Evarcha arcuate. Journal of Experimental Biology 206, 2733-2738. https://doi.org/10.1242/jeb.00478

[64] Wainwright Dylan, K., Kleinteich T., Kleinteich, A., Stanislav N. Gorb, Adam, P. Summers, Stick tight: suction adhesion on irregular surfaces in the northern clingfish, Biology letters 9.3 (2013): 20130234.

https://doi.org/10.1098/rsbl.2013.0234

[65] Edelmann, Jan ; Gärtner, Eric ; Eckert, Udo ; Griebel, Jan: Tool System for UV induced micro moulding of biomedical disposables.

In: Billington, D. ; European Society for Precision

Engineering and Nanotechnology -EUSPEN-:

18th International Conference of the European Society for

Precision Engineering and Nanotechnology, EUSPEN 2018.

Proceedings : June 4th-8th June 2018, Venice, IT

Bedford: Euspen, 2018, S.265-266

[66] Kugler, Till ; Blau, Peter ; Edelmann, Jan ; Eckert, Udo ; Koriath, Hans-Joachim ; Bogachev, Yuri P. ; Sakharova, Olga P.: Technologies for micro- and precision machining applications : Presentation held at International Specialized Exhibition Metalloobrabotka, 23.-27.05.2016, Moscow. 2016

[67] Schubert, Andreas ; Groß, Stefan ; Edelmann, Jan ; Eckert, Udo:

Manufacturing of micro fluidic moulds by combining micro milling and laser structuring.

In: Leach, R. ; European Society for Precision Engineering and Nanotechnology -EUSPEN-:

14th International Conference \& Exhibition of the European Society of Precision Engineering and Nanotechnology 2014 : 2nd June-6th June 2014, Dubrovnik, Croatia; EUSPEN 2014 Bedford: Euspen, 2014, S.97-101

[68] Shu, L.H., K. Ueda, I. Chiu, and H. Cheong. 2011. "Biologically Inspired Design.” CIRP Annals 60 (2): 673-93. https://doi.org/10.1016/j.cirp.2011.06.001. 